\title{
14
}

\section{RELIABILITY ESTIMATION OF TALL RC CHIMNEYS}

\author{
M.B.Krakovski
}

Department of Naval Architecture, University of Ulsan, Ulsan P.O.Box 18, Republic of Korea 680-749

[Permanently Leading Researcher, Research Institute of Concrete and Reinforced Concrete, Moscow, Russia]

A RC chimney $330 \mathrm{~m}$ high has been designed for a power plant in Kazakhstan. In the course of construction it was discovered that geometrical axis of the chimney had tipped out of vertical. As a result it was decided to reduce the chimney hight down to $300 \mathrm{~m}$. The paper deals with the investigation carried out in order to check whether the chimney $300 \mathrm{~m}$ high is sufficiently safe. The methodology of investigation and obtained results are presented.

\section{INTRODUCTION}

A RC chimney $330 \mathrm{~m}$ high has been designed for a power plant in Kazakhstan. In the course of construction it was discovered that geometrical axis of the chimney had tipped out of vertical. The maximum deviations of 65 $\mathrm{cm}$ were observed at elevations of 94 and $99.5 \mathrm{~m}$. As a result it was decided to reduce the chimney hight down to $300 \mathrm{~m}$.

The investigation under discussion has been carried out in order to evaluate whether the chimney $300 \mathrm{~m}$ high is sufficiently safe. With this aim in view reliabilities of two chimneys 300 and $330 \mathrm{~m}$ high were estimated and compared.

The investigation was ordered by a design office of power plant engineering. The office had at its disposal field measurements of concrete strength and of chimney geometrical dimensions. The measurements had been taken to control the quality of construction. These primary data were grouped and represented as histograms, which were taken to be probability density fucntions of corresponding basic variables.

According to the terms of the contract, in the course of reliability analysis all requirements of conventional design should have been met. Therefore it was decided to utilize an available computer program, which designers used for conventional analysis, though it took about 20 minutes to perform one deterministic analysis for specified values of basic variables.

The multitude of failure modes presented additional difficulties: as a result of each deterministic analysis the values of 8 output parameters were determined, i.e., 8 failure modes could occur.

All above peculiarities of the problem make it difficult, if not impossible, to analyse reliability of the chimneys using Level 2 (FORM/SORM) or 
importance sampling methods (see, e.g., [1-3]). Among other things, both these approaches require probability density functions of basic variables as well as limit state functions to be continuous. But probability density functions in the form of histograms are not continuous and 8 limit state functions can not be defined analytically in closed form: using conventional deterministic analysis it is possible only to check, whether the structure with specified values of basic variables fails or not.

In this connection in order to carry out reliability analysis, Monte Carlo simulation with subsequent approximation of the results by Pearson's curves [4] was used [5]. In the paper the methodology of investigation as well as obtained results are described.

\section{STATEMENT OF PROBLEM}

For reliability analysis strengths of concrete $S$ and reinforcement $R$ as well as geometrical dimensions of the chimneys (internal radius $r$ and thickness of the walls $h$ ) were taken as basic variables. The loads were assumed to be deterministic and equal to their design values. The analysis has shown that under this assumption the calcultions resulted in conservative estimates.

In the course of construction of the chimney $300 \mathrm{~m}$ high the following parameters were checked at 26 elevations: strength of concrete, internal radii of the chimney, wall thickness. Strength of concrete was checked in two ways - by compression tests of specimens and by non-destructive ultrasonic tests. The results of geodetic measurements as well as concrete tests were represented as histograms in chimney sections at 26 elevations. To take an example, in Fig. 1 are shown the histograms at an elevation of $130 \mathrm{~m}$ for internal radius (a), wall thickness (b) and concrete strength (c). Thus, a number of basic variables representing geometrical dimensions and concrete strength was 78 .

As to strength of reinforcement, it was dealt with in the following way. Ten different types of rebars (namely, 12, 16, 18, 20, 22, 25, 28, 32, 36, 40 $\mathrm{mm}$ in diameter) were used for chimney reinforcement. Since the rebars of different types are produced separately, they can be regarded as distinct materials. Therefore strengths of different types of rebars were assumed to be normally distributed statistically independent random variables with the same characteristics - mean values, $450 \mathrm{MPa}$, and standard deviations, $25 \mathrm{MPa}$. Design strength of reinforcment, $375 \mathrm{MPa}$, has an exceedance probability of 0.9986 . Thus, additional 10 basic variables representing strength of reinforcement were considered, making a total number of basic variables equal to 88 .

As a result of each deterministic analysis the following 8 output parameters were determined: stresses in concrete $\sigma_{c}$; stresses in vertical reinforcement $\sigma_{v}$ and hoop bars $\sigma_{h}$; vertical $a_{v}^{1}, a_{v}^{2}$ and horizontal $a_{h_{0}}^{1} a_{h}^{2}$ crack widths; deflection $f$ of the top of the chimney. Here superscripts 1 and 2 are related, respectively, to the upper part of the chimney $1 / 3 H$ high and to its lower part $2 / 3 H$ high ( $H$ is a total hight of the chimney).

The allowable values of crack widths are: $\left[a_{v}^{1}\right]=\left[a_{h}^{1}\right]=0.1 \mathrm{~cm} ;\left[a_{v}^{2}\right]=\left[a_{h}^{2}\right]$ $=0.2 \mathrm{~cm}$. The allowable deflections [f] of the top of the chimneys 300 and 330 $\mathrm{m}$ high are, respectively, 6.03 and $6.58 \mathrm{~m}$. The allowable values for stresses in concrete and reinforcement are discussed below.

All above numerical values are determined in accordance with Russian codes and standards regulating analysis and design of chimneys. 
a

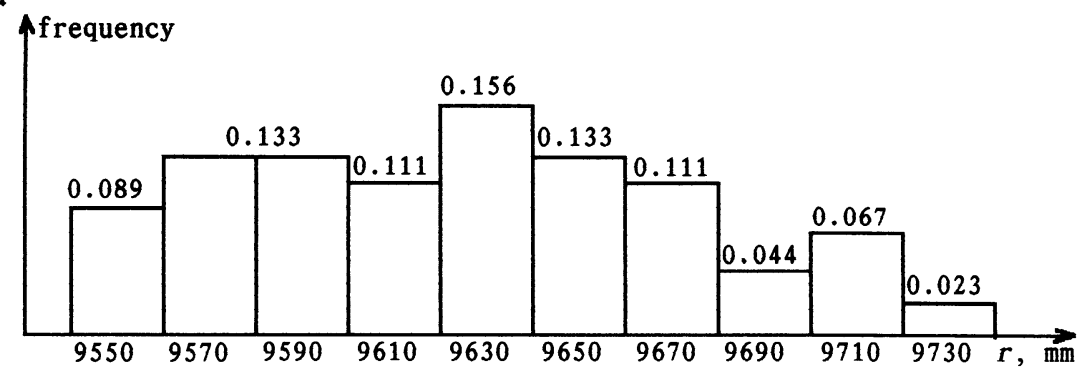

b

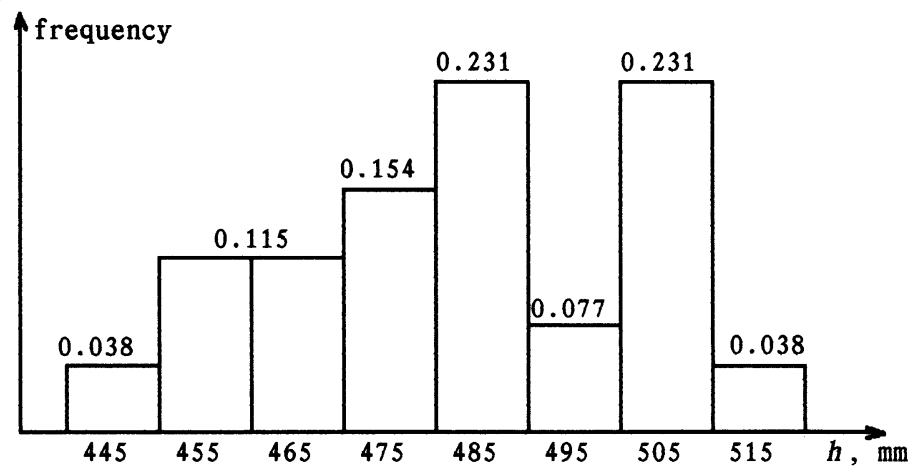

c

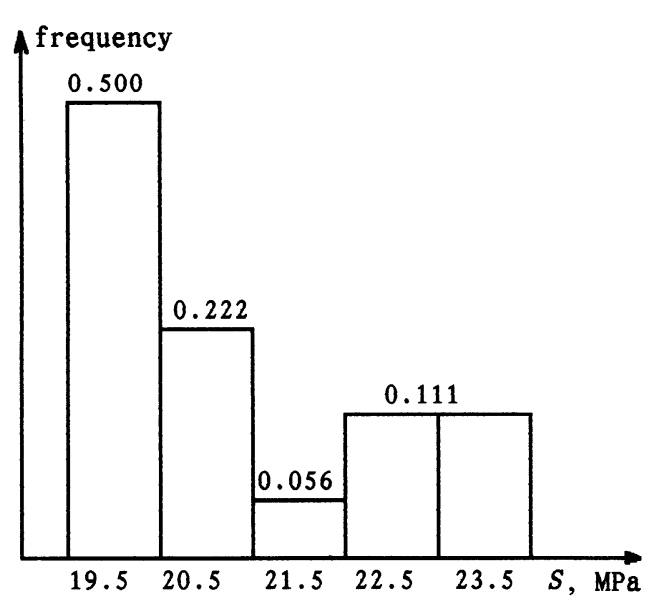

Fig. 1. Histograms 
Reliability of the chimneys was assumed to be the probability that output parameter values will be below their allowable values. Reliability was determined with respect to:

- each of the output parameters $\left(\sigma_{c}, \sigma_{v}, \sigma_{h}, a_{v}^{1}, a_{v}^{2}, a_{h}^{1} a_{h}^{2} f\right)$;

- strength limit state (failure occurs if at least one of the parameters $\sigma_{c}, \sigma_{v}$, $\sigma_{h}$ exceeds its allowable value);

- serviceability limit state (failure occurs if at least one of the parameters $a_{v}^{1}, a_{v}^{2}, a_{b_{b}}^{1} a_{b_{0}}^{2} f$ exceeds its allowable value);

- strength and serviceability limit states (total reliability, failure occurs if at least one of all output parameters exceeds its allowable value);

\section{SOLUTION OF PROBLEM}

The following algorithm was used to solve the problem.

1. According to specified probability density functions of basic variables obtain $m$ sets of their random realizations ( 88 realizations in each set).

2. Carry out $m$ deterministic analyses of the structure by the conventional method. As a result obtain $m$ sets of 8 output parameters.

3. Choose one value representing each set of the output parameters. This item is discussed in detail later.

4. For $m$ values, obtained at step 3 , choose a suitable probability density function $y(z)$ out of the family of Pearson's curves.

5 . Determine reliability of the structure by numerical integration

$$
P=\int_{u_{1}}^{u_{2}} y(z) d z
$$

The ways of specifying the values of $u_{1}, u_{2}$ are described below.

Now let us discuss step 3 of the algorithm, when reliability analysis is carried out with respect to different combinations of the output parameters. Consider first the case of reliability estimation with respect to one parameter $\sigma_{v}$. Ten random realizations $R_{i}^{k}(i=1, \ldots, 10)$ of strength of rebars of 10 different above mentioned types are used as input data for $k$ th deterministic analysis. As a result of this analysis 26 values of stresses in vertical reinforcement $\sigma_{v j}^{k}(j=1, \ldots, 26)$ are obtained at 26 sections of the chimney. At each section the minimum

$$
\min _{i}\left(R_{i}^{k}-\sigma_{v j}^{k}\right)
$$

is found and then minimum $\sigma_{v}^{k}$ of these minima among all 26 sections is determined

$$
\sigma_{v}^{k}=\min _{j} \min _{i}\left(R_{i}^{k}-\sigma_{v j}^{k}\right)(k=1, \ldots, m)
$$

At this point step 3 of the algorithm is completed. At step 4 the values of $\sigma_{v}^{k}$ are approximated by a suitable Pearson's curve $y(z)$. The structure is safe if and only if $\sigma_{v}^{k} \geq 0$. Therefore the integration by eqn. (1) is performed with $u_{1}=0, u_{2}=+\infty$. 
In much the same way reliability with respect to each of other output parameters $\left(\sigma_{c}, \sigma_{h}, a_{v}^{1,} a_{h}^{1}, a_{v}^{2}, a_{h}^{2}, f\right)$ was determined.

In order to check the calculated values of reliabilities, another approach was used as well. Instead of minima calculated in accordance with eqn. (2), the following maxima were determined:

$$
\max _{i} \frac{\sigma_{v j}^{k}}{R_{i}^{k}}
$$

and then maximum $\sigma_{v 1}^{k}$ of these maxima among all 26 sections was determined - eqn. (5) was used instead of eqn. (3):

$$
\sigma_{v 1}^{k}=\max _{j} \max _{i} \frac{\sigma_{v j}^{k}}{R_{i}^{k}}
$$

Thereupon again a suitable Pearson's curve is chosen and integration by eqn. (1) is performed with $u_{1}=-\infty, u_{2}=1$.

Now consider reliability evaluation with respect to strength limit states, i.e., three output parameters $\sigma_{c}, \sigma_{v}$ and $\sigma_{h}$ are taken into account. In this case the values $\sigma_{c 1}^{k}$ and $\sigma_{h 1}^{k}$ similar to $\sigma_{v 1}^{k}$ are determined:

$$
\begin{aligned}
& \sigma_{c 1}^{k}=\max _{j} \frac{\sigma_{c j}^{k}}{S_{j}^{k}} \quad(k=1, \ldots, m) \\
& \sigma_{h 1}^{k}=\max _{j} \max _{i} \frac{\sigma_{h j}^{k}}{R_{i}^{k}} \quad(k=1, \ldots, m)
\end{aligned}
$$

and then

$$
\begin{aligned}
& \sigma^{k}=\max \left(\sigma_{c 1}^{k}, \sigma_{v 1}^{k}, \sigma_{h 1}^{k}\right)=\max \left[\left(\max _{j} \frac{\sigma_{c j}^{k}}{S_{j}^{k}}\right),\left(\underset{j}{\max } \max _{i} \frac{\sigma_{v j}^{k}}{R_{i}^{k}}\right),\right. \\
& \left.\left(\max _{j} \max _{i} \frac{\sigma_{h j}^{k}}{R_{i}^{k}}\right)\right] \quad(k=1, \ldots, m)
\end{aligned}
$$

is determined, representing the outcome of the $k$ th simulation. Here $S_{j}^{k}$ is a realization of concrete strength at $j$ th section $(j=1, \ldots, 26)$ in $k$ th simulation $(k$ $=1, \ldots ., m)$. The values of $S_{j}^{k}$ are used as input data for each deterministic design. The limits of integration in eqn. (1) are $u_{1}=-\infty, u_{2}=1$.

Reliability with respect to serviceability limit states as well as total reliability (with respect to all output parameters) is calculated in much the same manner. Take, for example, the total reliability. The outcome of each simulation is represented by the value: 


$$
t^{k}=\max \left[\left(\max _{j} \frac{\sigma_{c j}^{k}}{S_{j}^{k}}\right),\left(\max _{j} \max _{i} \frac{\sigma_{v j}^{k}}{R_{i}^{k}}\right), \ldots,\left(\max _{j} \frac{a_{h j}^{k}}{\left[a_{h}^{2}\right]}\right), \frac{f^{k}}{[f]}\right]
$$

Then a suitable Pearson's curve $y(z)$ is chosen for $t^{k}$ and integration between limits $u_{1}=-\infty$ and $u_{2}=1$ is performed.

As was indicated above, it took about 20 minutes to carry out one deterministic analysis. Therefore several computers working simultaneously were used.

In order to determine necessary sample size for reliability analysis, the values of reliabilities were computed after every 500 simulations. The results for sample sizes 2000,2500,3000 appeared to be closely allied. Therefore it was decided to consider results with $m=3000$ as final ones.

By way of illustration in Table 1 are presented random realizations of basic variables $r, h, S$ at an elevation of $130 \mathrm{~m}$ obtained from histograms in Fig. 1, and in Table 2 are given the values of some output parameters at the same elevation. All data are listed for the first 5 simulations. As can be seen from the Tables, it was not clear in advance, which of the chimneys was more reliable.

Below are given some of the Pearson's curves, approximating results of Monte Carlo simulations. For values of $\sigma_{c}^{k}$

$$
\sigma_{c}^{k}=\min _{j}\left(S_{j}^{k}-\sigma_{c j}^{k}\right) \quad(k=1, \ldots, m)
$$

in chimneys 300 and $330 \mathrm{~m}$ high Pearson's curves turned out to be, respectively, of types 7 eqn. (11) and 4 eqn. (12):

$$
\begin{aligned}
y(z)= & 0.2498\left[1+\left(\frac{z-3.402}{0.2315}\right)^{2}\right]^{-2.882} \\
y(z)=0.2753\left[1+\left(\frac{z-1.460}{0.2096}\right)^{2}\right]^{-11.04} & \times \exp \left[-11.04 \arctan \left(\frac{z-1.460}{0.2069}\right)\right]
\end{aligned}
$$

For values $\sigma_{v}^{k}$ (3) in chimneys 300 and $330 \mathrm{~m}$ high Pearson's curves turned out to be of type 2 :

$$
\begin{aligned}
& y(z)=0.1418\left[1-\left(\frac{z-157.7}{107.75}\right)^{2}\right]^{-2.111} \\
& y(z)=0.1307\left[1-\left(\frac{z-158.0}{61.31}\right)^{2}\right]^{-1.227}
\end{aligned}
$$


Table 1

Realizations of basic variables at an elevation of $130 \mathrm{~m}$

\begin{tabular}{cccc}
\hline Simulation No & $r, \mathrm{~mm}$ & $h, \mathrm{~mm}$ & $S, \mathrm{MPa}$ \\
\hline 1 & 9690 & 475 & 19.5 \\
2 & 9570 & 465 & 19.5 \\
3 & 9570 & 495 & 19.5 \\
4 & 9630 & 505 & 20.5 \\
5 & 9610 & 505 & 25.5 \\
\hline
\end{tabular}

Table 2

Results of Monte Carlo simulation at an elevation of $130 \mathrm{~m}$

\begin{tabular}{|c|c|c|c|c|c|c|c|c|c|c|}
\hline \multirow{4}{*}{$\begin{array}{l}\text { Simu- } \\
\text { lation } \\
\text { No }\end{array}$} & \multicolumn{6}{|c|}{ Stresses, MPa } & \multirow{2}{*}{\multicolumn{2}{|c|}{$\frac{\text { Crack width }}{a_{v}^{2}}$}} & \multirow{2}{*}{\multicolumn{2}{|c|}{$\frac{\times 10^{3}, \mathrm{~cm}}{a_{h}^{2}}$}} \\
\hline & \multicolumn{2}{|c|}{$\sigma_{c}$} & \multicolumn{2}{|c|}{$\sigma_{v}$} & \multicolumn{2}{|c|}{$\sigma_{h}$} & & & & \\
\hline & \multicolumn{10}{|c|}{ Chimney hight $H=$} \\
\hline & $300 \mathrm{~m}$ & $330 \mathrm{~m}$ & $300 \mathrm{~m}$ & $330 \mathrm{~m}$ & $300 \mathrm{~m}$ & $330 \mathrm{~m}$ & $300 \mathrm{~m}$ & $330 \mathrm{~m}$ & $300 \mathrm{~m}$ & $330 \mathrm{~m}$ \\
\hline 1 & 11.0 & 9.7 & 193 & 192 & 253 & 276 & 75 & 73 & 74 & 72 \\
\hline 2 & 11.2 & 8.9 & 190 & 189 & 250 & 253 & 72 & 71 & 71 & 70 \\
\hline 3 & 10.6 & 8.6 & 197 & 197 & 260 & 259 & 79 & 87 & 78 & 83 \\
\hline 4 & 10.7 & 8.6 & 200 & 218 & 263 & 263 & 83 & 66 & 80 & 85 \\
\hline 5 & 10.9 & 10.3 & 200 & 217 & 263 & 222 & 83 & 78 & 80 & 77 \\
\hline
\end{tabular}

The results of the investigation were as follows. Probabilities of failure of both chimneys with regard to each of the output parametets $\sigma_{c}, \sigma_{v}, \sigma_{h}$ as well as with regard to strength limit state were less than $10^{-7}$. Probabilities of failure of both chimneys with regard to each of the output parameters $a_{v}^{1}, a_{v}^{2}$, $a_{b_{5}}^{1} a_{b_{0}}^{2} f$ as well as with regard to serviceability limit state were less than $10^{-5}$. Reliabilities estimated according to equations of type (3) and (5) with respect to the same output parameter were in close agreement. The total failure probabilities of chimneys 300 and $330 \mathrm{~m}$ high were, respectively, $8.184 \times 10^{-7}$ and $3.166 \times 10^{-6}$. All failure probabilities are small enough. Failure probabilites of chimney $300 \mathrm{~m}$ high did not exceed those of chimney $330 \mathrm{~m}$ high. On the basis of these results it was concluded that the chimney $300 \mathrm{~m}$ high was sufficiently safe. The chimney is in service now.

\section{CONCLUSION}

A rather complex practical problem was solved: reliabilites of two RC chimneys - $300 \mathrm{~m}$ high (with deviations of geometrical axis) and $330 \mathrm{~m}$ high (without the deviations) - were estimated. Results of field measurements in the form of histograms were used as probability density functions of basic variables. Eight failure modes of the chimneys could not be defined analytically in closed form. Therefore it was very difficult, if not impossible, 
to use Level 2 (FORM/SORM) or importance sampling methods for reliability analysis. To solve the problem Monte Carlo simulation with subsequent approximation of the results by Pearson's curves was applied. The methodology of investigation was developed and checked for accuracy. Calculations were performed, reliabilities of two chimneys with respect to different combinations of output parameters were determined and compared with each other. On the basis of the obtained results it was concluded that the chimney $300 \mathrm{~m}$ high was sufficiently safe. The chimney is in service now.

\section{REFERENCES}

1. P.Thoft-Christensen and M.J.Baker, Structural Reliability Theory and its Applications, Springer, New York, 1982.

2. M.Hohenbichler, S.Soltwitzer, W.Kruse and R.Rackwitz, New light on firstand second-order reliability methods, Structural Safety, 4, 1987, 267.

3. S.Engelung and R.Rackwitz, A benchmark study on importance sampling technique in structural reliability, Structural Safety, 12, 1993 255.

4. W.P.Elderton and N.L.Johnson, Systems of Frequency Curves, Cambridge University Press, 1969.

5. M.B.Krakovski, Improvement of Russian Codes for RC Structures Design on the Basis of Reliability Theory, Proceedings of IFIP WG7.5 Fifth Working Conference on Reliability and Optimization of Structural Systems, Japan, March, 1993, North-Holland, 141. 